\title{
Lanzhou Beef Noodles and Mechanical Process of Dough Machine Extrusion
}

\author{
He Wei $^{1}$, Li Wanxiang ${ }^{2}$, Zhai Chaokai ${ }^{3}$ \\ ${ }^{1}$ School of Art and Design, Lanzhou Jiaotong University, Lanzhou, Gansu, China \\ ${ }^{2}$ School of Mechanical and Electrical Engineering, Lanzhou Jiaotong University, Lanzhou, Gansu, China \\ ${ }^{3}$ School of Mechanical and Electrical Engineering, Lanzhou Jiaotong University, Lanzhou, Gansu, China
}

Keywords: Lanzhou beef noodles, Dough machine, Twin screw, Mechanical process

\begin{abstract}
With the development of modern food processing industry, processing machinery has been has developing and improving. In-times to special the special s a case-like high water content (about $48 \%$ ) in Lanzhou beef noodles, this thesis centers on the report of the report of the specified mechanical and electrical product of the producing flour. The twin-screw extruder of the dough, designed on a optimal basis, is mainly ed of extrusion mechanism, repulsion mechanism, and drive mechanism, able to complete the process of stirring, fermentation, kneading, pounding, ramen, etc. meeting the changing requirements of markets and processing.
\end{abstract}

\section{Introduction}

Lanzhou beef noodles is a century-old accumulation of food culture, as one of the three Chinese-style fast food pilot promotion varieties, it is known as "The first noodle of China." According to incomplete statistics, Lanzhou beef noodle business has reached tens of thousands across the country, In Lanzhou alone, there are more than 1200 large and small beef noodle restaurants, with over 10,000 employees, The daily consumption is more than 500,000 people-times, and the industry's annual output value exceeds 700 million yuan. However, the low threshold of Lanzhou beef noodles, relatively simple investment, poor sanitation, and a chaotic business environment have led to Lanzhou beef noodles that have not been able to become a strong brand, although they have traveled all over the country.

In the city's fast-paced life, people are increasingly demanding the speed and hygiene of beef noodles. However, most domestic beef noodle restaurants still use traditional hand-made noodles, kneading noodles, and ramen. Not only is it inefficient, but the level of the noodle, kneading, and ramen masters will also cause the taste of the noodles to be unstable, As a result, the level of ramen was uneven, which seriously affected the reputation of Lanzhou beef ramen in the field ${ }^{[1]}$.

The food machinery industry has been greatly developed, and many traditional food industries have introduced modern food machinery to reduce operating costs ${ }^{[2]}$. However, there is currently no machine in China that can efficiently export beef ramen noodles. Therefore, it is urgent to design and promote a high-efficiency, high-quality beef noodle dough machine.

\section{Design}

Under the action of pressure, food materials pass through the mold to produce mature or semi-cooked, puffed or non-puffed food in a directional and continuous molding, which is called " Squeeze food “. Squeeze molding is characterized by low cost, large production capacity, various product shapes, high energy efficiency, high product quality, almost no loss of food raw materials, and easy production of new products. For the preliminary production of beef noodle, three special-purpose machines with different transmission types were designed for roller-type dough machine, torsion face bar machine and twin screw extrusion face bar machine.

\subsection{Roller-Type Dough Machine}

The roller-type dough machine is a kind of mechanical equipment that realizes the extrusion of 
noodle sticks by simulating the manual rolling process. The working principle of the device is shown in Figure 1, The left half is a transmission device composed of a gear train to realize the conversion and transmission of power. The right half is a roller, which is used to simulate the basic function of a rolling pin during manual rolling. The left and right parts are connected by a connecting rod. The motive power output by the motor drives the output gear to rotate through the transmission device. The output gear drives the drum to reciprocate on the support plate with multiple grooves through the connecting rod. Into the groove, in order to realize the extrusion molding of noodles.

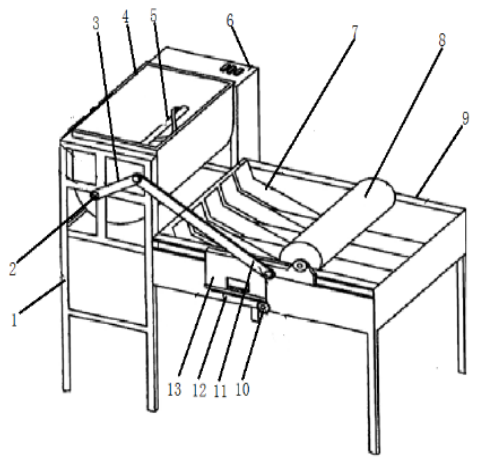

Fig.1 Structure Diagram of Rotary Drum Type Dough Machine

3. Bracket; 2. Clutch; 3. Driving Crank;4. Noodle Buckets; 5. Agitators; 6. Two-Stage Deceleration Device; 7. Flip the Plate; 8. Rollers; 9. Face Box; 10. Pinion; 11. Connecting Rods; 12. Racks; 13.

Slider

The roller-type dough machine can effectively reduce the labor of repeated pulling, pounding, kneading, rubbing etc. in the process of manual and flour gluten. By reducing the workload of workers, labor costs will be reduced, labor intensity will be further reduced, and labor efficiency will also be improved. However, in the process of forming noodle sticks, the dough was only repeatedly lifted and flattened in one direction, and the noodle mixing effect was not uniform, The noodles made are not strong enough.

\subsection{Torsion Face Bar Machine}

Using power decomposition technology, equidistant reducing screw design technology and planetary gear train torsion mechanism, full-truth simulation of the hand-crafted process, After the dough is kneaded and smoothed by the machine, it is extruded into 408 coarse noodles. Pull operation to improve the ductility of noodles. The torsion face bar machine is composed of thrust mechanism, force mecha-nism, planetary gear train and other important parts (Figure 2).

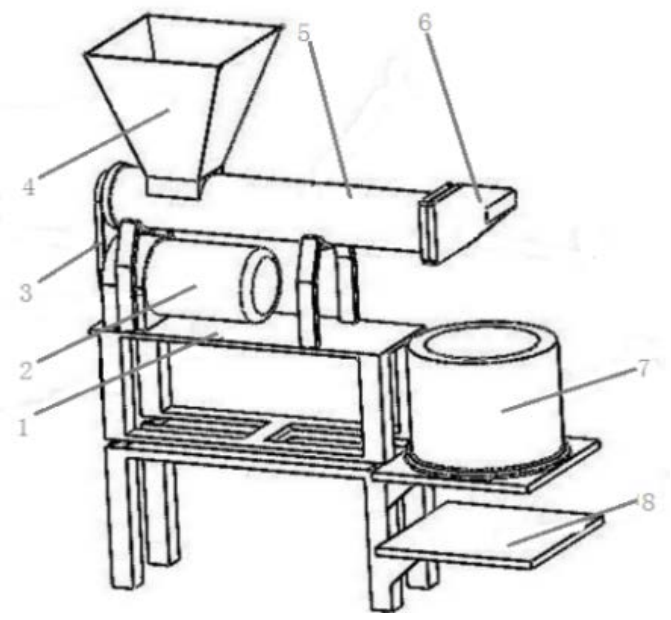

Fig.2 Schematic Diagram of Rotary Noodle Dough Machine

1. Panels; 2. Motor; 3. Sprockets; 4. Hopper; 5. Kneading the Body; 6. Die Hole Plate; 7. Pulling the Agency; 8. Mesa.

When the motor is connected to the secondary reducer through a sprocket, one end of the 
secondary reducer is connected to the planetary gear train, and the other end is connected to the kneading mechanism of the dough stick machine through a V-belt. Because they are connected to the same reducer, the torque obtained is equal, and the instability caused by the unequal torque will not occur ${ }^{[4]}$. The two moments respectively drive the planetary gear train and the screw in the kneading part. The dough is put into the hopper first. During the rotation of the screw, the dough is pressed by the screw between the screw and the barrel, and is pushed into the planetary gear train with the rotation of the screw. Due to the rotation of the planetary gear train, the dough is more twisted. It is malleable.

Use one motor to drive two parts to move at the same time to make the system more stable. The use of equidistant reducer screws ensures repeated kneading of the dough so that the noodles and water molecules are fully fused to ensure the taste of the noodles. However, the screw is easily heated during the work.

\subsection{Twin Screw Extrusion Face Bar Machine}

The research shows that the twin screw extrusion technology develops rapidly because the material can be fully mixed and kneaded completely. In addition, the twin screws engage each other and have a self-cleaning function, which avoid the phenomenon of coking on the surface of the sleeve caused by the screw plugged material that often occurs in single screw extruder ${ }^{[7]}$. Wang Hongwu and others used a new twin-screw food extruder to conduct research on soybean protein and engineering meat products ${ }^{[8]}$. Zhang Bing-wen, Qi Guo-dong etc. successfully developed high-protein breakfast nutrition powder using twin-screw extrusion technology ${ }^{[9]}$.

The use of twin screw extrusion machine processing beef ramen agent. At the rated torque, the squeeze pressure between the twin screws, between the screw and the barrel is sufficient to bring the extruded surface to an artificial level.

The twin screw extrusion face bar machine consists of a drive mechanism, a thrust mechan- ism and an extrusion mechanism (figure3).

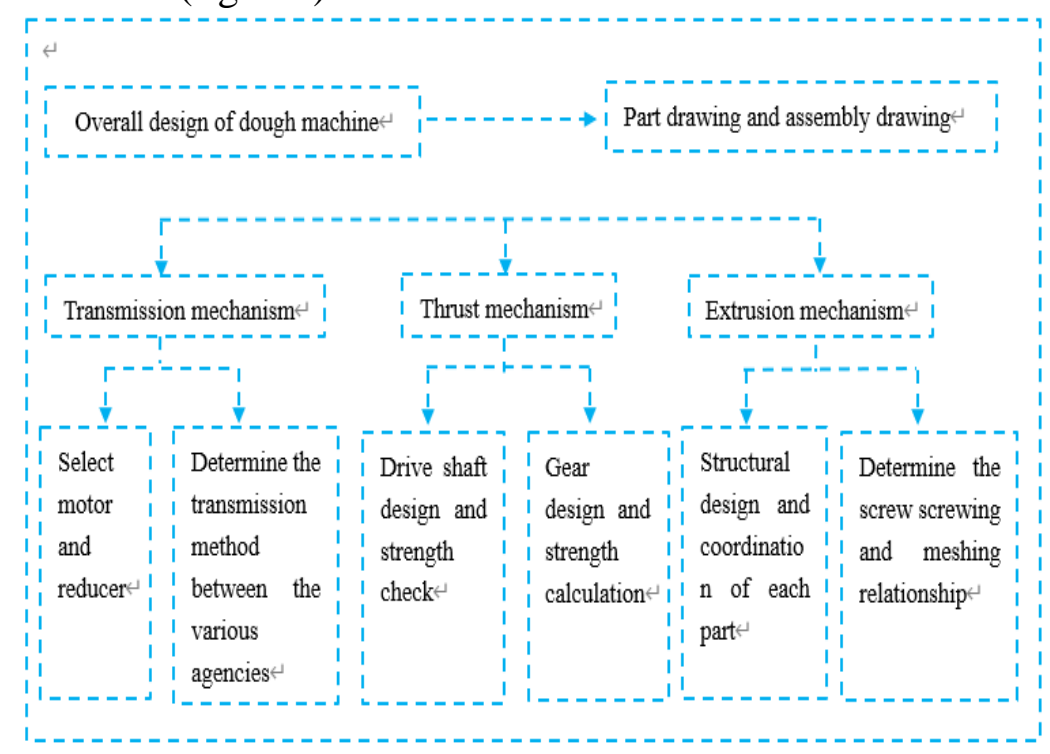

Fig.3 Principle Diagram of Twin Screw Extrude

The main components of the transmission consist of 2.2kw AC motor, WPA-80 reducer, belt transmission, chain transmission and gear transmission. The main components of the kneading part include a twin-screw screw propelling mechanism, a face die and an additional fabric bucket. The pulling and twisting part is mainly composed of gear transmission device, gear planetary gear train device and pulling and twisting device components (figure 4). 


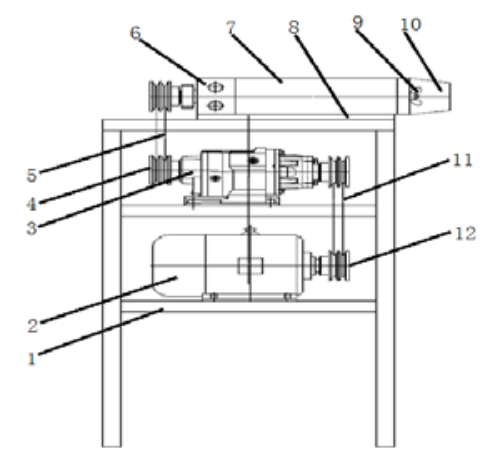

Fig.4 Structure Diagram of Twin Screw Extrude

1. Racks; 2. Motor; 3. Decelerator; 4. Sprockets; 5. Chain; 6. Housing; 7. Barrel; 8. Panels; 9.

Butterfly Nut; 10. Cone; 11 . V-Belt; 12. with Wheels;

When the motor is driven and the traction $\mathrm{V}$ belt is driven, the reducer is connected to the drive shaft through a sprocket. There is a pair of conjugate gears between the two shafts. Because a screw is connected to each end of the two shafts, the two screws are driven to rotate. When the dough is put into the hopper, the screw pushes the dough forward, and through extrusion, the dough is finally passed through the tapered exit to obtain a shaped noodle stick.

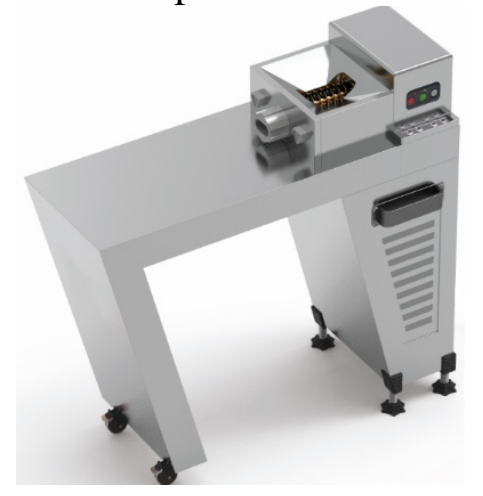

Fig.5 Prototype of Twin Screw Extrude

Type dough machine

The twin screw extrusion stick machine has been repeatedly tested and has been successfully manufactured. The prototype is shown in figure 5. This machine is currently the only one in China that specializes in the process of beef ramen with high water content and kneading, pounding, shaking, smoothing and sticking out during the production process. It is mainly manual, high labor intensity, low efficiency, poor sanitation, Special kitchen electromechanial products designed for high salary requirement It is mainly used in pasta processing places such as beef ramen restaurants. After testing, it can replace the work of three kneading dough gluten chefs.

\section{Design of Extrusion Mechanism and Stress Analysis}

\subsection{Twin Screw Design}

The twin screw is the core equipment of the extrusion mechanism. It is the most important part for conveying and kneading the dough. Its structural performance will directly affect the working state and working efficiency of the extrusion mechanism.

Due to the high flexibility requirements of the output surface agent, the dough cannot generate high heat when it is conveyed and kneaded in the twin screw, and it should be as close as possible to room temperature (about $30^{\circ} \mathrm{C}$ ). Due to the comprehensive consideration of the high temperature of the dough when the dough is transported and kneaded, which is not conducive to the phenomenon of stranding caused by conveying, the twin screw is designed as a gradient ordinary screw, and the cross section of the screw is designed as a semicircular shape.

(1) Choice of twin screw material 
The screw is subject to a large torque during operation and runs at normal temperature. Due to the working relationship between the screw and the barrel, mechanical friction, wear, scartc- hes etc. will be lost. As a result, the screw may have severe wear and break, as well as the increase in the barrel gap to reduce the yield and other forms of failure.

According to the actual working conditions of the screw in the noodle stick machine, under the condition that the screw can work normally, we must choose the appropriate material. Because H62 (that is, four or six brass: ordinary brass drawn rods, semi-rigid, $40-80 \mathrm{~mm}$ ) has good mechanical properties, good plasticity under cold and hot conditions, good machinabi- lity, easy brazing and welding, and corrosion resistance, Select H62 as the material of the screw.

(2) Screw design calculation

Spiral height: $H=248 \mathrm{~mm}$

Screw diameter: $D=59 \mathrm{~mm}$

Pitch: $I=41.33 \mathrm{~mm}$

Number of coils: $R=6 \mathrm{~mm}$

Distance between adjacent slots: $B=43 \mathrm{~mm}$

Screw axial width: $b=4.3 \mathrm{~mm}$

The center of the conch round centule screw: $L=34.5 \mathrm{~mm}$

Slot radius: $r=20 \mathrm{~mm}$

Considering that the installation of the twin screws is uniformly mixed with the processing of the fabric, and to avoid interference when the two screws rotate, and determines that the center distance between the two screws is $60 \mathrm{~mm}$.

(3) The strength of the screw

The consumable part in a twin screw extruder is the screw. Therefore, it is necessary to calcul- ate the strength of the screw. Here we need to determine the following parameters: the axial force of the screw FZ, the maximum pressure at the head $\mathrm{P}$, and the torque Mt at the screw.

\subsection{Twin Screw Stress Analysis}

Stress analysis of the key components of the dough rod machine. In the extrusion mechanism, the driving end of the screw is analyzed, and in the thrust mechanism, the contact portion of the shaft with the gear, sprocket, key and other components is subjected to stress analysis.

(1) Determine the base axis: Select the base screw cylinder face in the reference geometry and insert the screw base shaft.

(2) Generating static studies: establishing new studies for stress analysis.

(3) Define material properties: Select the screw material as H62, i.e. ordinary brass.

(4) Add constraints: select the advanced clamp, select the cylindrical face inside the screw, determine the circular translation, and the Simulation constraint round shift.

(5) Applied torque: The face of the selected torque is the connecting face between the screw and the pin shaft, the reference direction takes the base shaft 1 , and the torque value is $95.5 \mathrm{~N} \cdot \mathrm{m}$.

(6) Force force: The centrifugal force of the screw load selects the reference shaft as the reference shaft and the angular speed is $10.47 \mathrm{rad} / \mathrm{s}$.

(7) Divide the grid and run: Select the default grid parameters in the grid to generate the grid as shown in Figure 6.

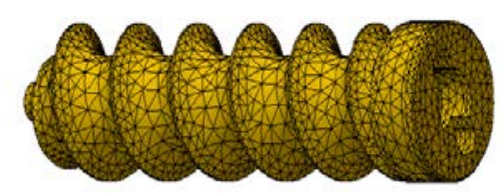

Fig.6 Grid Diagram of Screw

The grid cell size is 3.78 , the ratio is 1.5 , and the solve analysis is run. 


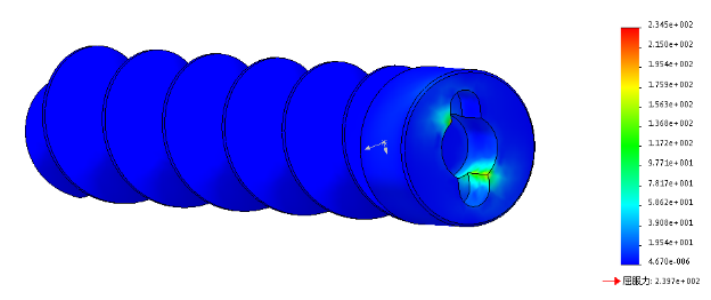

Fig.7 Stres Diagrams of Screw

The vonMises stress distribution of the screw is shown in figure 7. The maximum stress occurs at the contact surface between the screw and the pin. The maximum stress is $234.5 M P a$, which is less than the material's yield strength of $239.7 \mathrm{MPa}$, which meets the material,mechan- ical strength requirements.

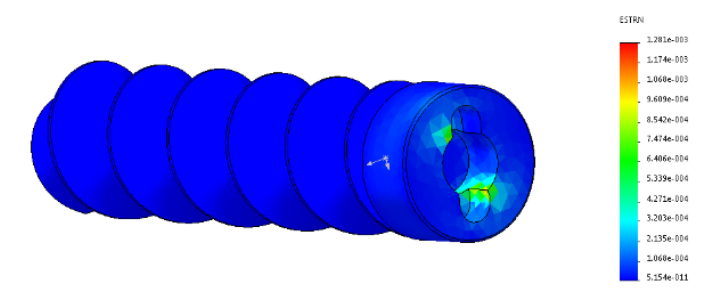

Fig.8 Strain Diagram of Screw

The vonMises of the screw should be distri- buted in figure 8 and meet the mechanical design requirements.

\section{Conclusions}

Using the equidistant variable-diameter scr-ew, realize the repeated kneading of the opposite group, so that the surface and water molecules fully blend, squeeze, so that the extension and elasticity of gluten protein maximize, to ensure the taste of the noodles. The use of planetary wheel system to simulate the process of manual kneading, due to the continuous twisting of the planetary rotation system to achieve the opposite group twisting and stretching, so that the dough more elastic, but also improve the ductex's ductility.

The twin-screw extrusion type noodle stick machine is a food processing machine designed for the current processes of beef ramen neutralizing noodles, awake noodles, kneading noodles, pounding noodles, dumping noodles and gluten. It significantly shortens the pre-production time of beef ramen, improves the efficiency of beef ramen production, and the use of this machine can greatly improve the sanitary condition of beef ramen production, can also save manpower, and reduce the operating cost of Lanzhou ramen restaurant. The future is a must-have product for Lanzhou ramen restaurants. It has strong market competitiveness and large market capacity, and is worth developing and producing. On the one hand, this product can free the ramen chef from heavy physical labor, improve production efficiency, reduce produc- tion costs, and achieve standardized and rapid continuous production; on the other hand, it can also greatly improve the hygienic status of the beef ramen production process and make beef ramen more hygienic and healthy.

Noodle stick machine extrusion machining in the value realization emphasizes from the traditional product manufacturing as the core, to provide a rich service content of products and relying on the service transformation, until to provide users with a more complete solution.

\section{References}

[1] Li Wanxiang, He Wei. Beef noodle and gluten smoothing machine [P].China, Utility Model Patent, 201020563501.8,2011 05 04.

[2] Cao Xiaohong. Analysis of composition changes and key technologies after high pressure treatment of foods [J]. Chinese Journal of Food Science, 2011, 9 (11): 26-31. 
[3] Yang Xiaoling, Deng Li, Dong Nan, Li Qiuping. Study on Twin-screw Extrusion Process of Reconstituted Beef Jerky [J]. Food and Machinery, 2013, 4 (29): 107-111.

[4] Gu Zhengbiao, Wu Shuanglan, Xu Yihu. Application of Extruder in Starch Deep Processing [J]. Grain and Feed Industry, 2000, 4: 42-44.

[5] Wei Yimin, Zhang Bo, Chen Fengliang. Theory and Technology of Food Squeezing [M]. Beijing: China Light Industry Press.

[6] Jin Zhengyu, Xie Zhengjun, Zhao Jianwei, Shen Yu. Extruded food [M]. Beijing: China Light Industry Press.

[7] Yang Tao, Xin Jianmei, Xu Qing, Luo Hongyu. Status of research and application of twin screw extrusion technology in food industry [J]. Journal of Food and Biotechnology, 2009, 6 (28): 733-740.

[8] Wang Hongwu, Zhou Jianguo, Lin Bingjian. Effect of Process Parameters of Twin Screw Extruder on Tissue Protein [J]. Journal of the Chinese Cereals and Oils Association, 2001, 16 (2): 54-57.

[9] Zhang Bingwen, Qi Guodong, et al. Study on the production process of high protein breakfast nutrition powder using twin screw extrusion technology [J]. Journal of the Chinese Cereals and Oils, 2000, 15 (6): 58-62.

[10] Teng Qingming. Scheme comparison and strength analysis of transmission system of twin screw extruder [D]. Beijing University of Chemical Technology, 2008.

[11] Zhang Pingliang. Development and application of new food twin screw extruder in developed countries [J]. Food Industry, 2011, 12 (32): 89-90. 